\section{Manifeste de la SSP et de la SSPPEA concernant le TarMed}

Société Suisse de Psychiatrie et Psychothérapie, Société Suisse de Psychiatrie et Psychothérapie d'enfants et d'adolescents

\author{
Moins de 200 francs l'heure - Jamais! \\ Non à un tarif inférieur à l'actuel tarif \\ AVS/AI/AM/SUVA!
}

Pour la Société Suisse de Psychiatrie et Psychothérapie et la Société Suisse de Psychiatrie et Psychothérapie d'enfants et d'adolescents, le TarMed est inacceptable dans sa forme actuelle. Nous demandons des mesures concrètes, propres à réaliser les objectifs initiaux de la révision tarifaire pour la psychiatrie:

- adaptation aux autres revenus médicaux;

- évaluation correcte de son travail spécifique;

- couverture du besoin de rattrapage dans le domaine de l'infrastructure.

Correspondance:

Dr Hugo Ineichen

Membre du comité de la SSP

Rue des Métiers 4

CH-1008 Prilly
Pendant des années, la SSP et la SSPPEA ont cherché par la voie pondérée à faire intégrer dans le TarMed ces objectifs de la révision tarifaire convenus à l'origine. En vain!

Nous allons présenter sous peu un modèle de tarif conforme aux objectifs reconnus, dont la structure se fonde sur celle du TarMed.

Nous sommes bien conscients du fait que l'intégration de notre modèle de tarif dans le TarMed nécessite des négociations et du temps. Jusqu'alors nous attendons des mesures d'urgence qui assurent aux psychiatres un tarif horaire minimum (= chiffre d'affaires, non pas revenu) de 200 francs.

A cet effet, la SSP et la SSPPEA attendent de la FMH et de son Président:

- qu'ils prennent officiellement position, déclarant que la version actuelle du TarMed est inadéquate pour la psychiatrie et doit être modifiée aussitôt que possible;

- qu'en qualité d'association faîtière, ils interviennent avec détermination pour obtenir des autorités et des caisses-maladie les mesures d'urgence demandées.

Dans la situation actuelle du TarMed, la SSP et la SSPPEA se considèrent comme dupées. Le revenu des psychiatres n'atteint que la moitié du revenu médical moyen. Le TarMed réduit même les tarifs horaires en dessous du tarif AI actuellement en vigueur. La SSP et la SSPPEA vivent ainsi une grave crise de confiance envers l'association faîtière des médecins comme ils doutent aussi de la solidarité des autres groupes de spécialistes.

Les arguments de ce manifeste et l'état des faits sont présentés en détail dans la "Compilation: problèmes TarMed en psychiatrie» (juin 2000) de la Commission permanente des tarifs de la SSP/SSPPEA. 